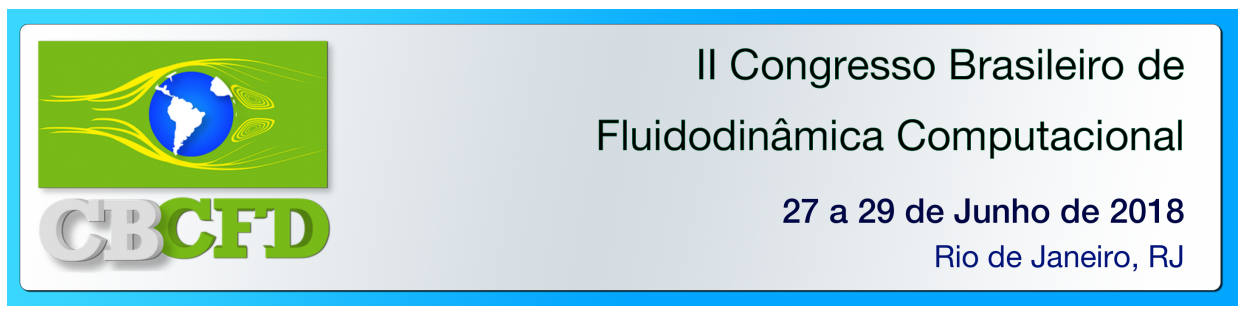

\title{
IMPLEMENTAÇÃO DE MODELOS DE ESCOAMENTO REATIVO EM MEIOS POROSOS NO OPENFOAM.
}

\author{
P. R. S. DA COSTA JR ${ }^{1,2}$, A. O. S. MORAES ${ }^{1}$, R. A. C. DIAS ${ }^{1}$, F. G. $\operatorname{COSTA}^{3}$, C. \\ SPEGLISH $^{3}$ \\ ${ }^{1}$ WIKKI BRASIL Consultoria em Engenharia LTDA \\ ${ }^{2}$ Universidade Federal do Rio de Janeiro, Escola de Química \\ ${ }^{3}$ CENPES-PETROBRAS \\ Email para contato: paulo.costa@wikki.com.br
}

\begin{abstract}
RESUMO - Dois modelos para escoamentos reativos em meios porosos foram implementados usando técnicas de Fluidodinâmica Computacional, visando aplicações de acidificação de reservatório carbonáticos do pré-sal e pós-sal. Os dois modelos utilizam o Modelo de Duas Escalas Contínuas, sendo que o primeiro é baseado na equação de Darcy para transporte de quantidade de movimento linear em meio poroso. No segundo, é proposto modelar a equação de conservação de quantidade de movimento linear usando a teoria da média volumétrica, levando em conta os efeitos inerciais e difusivos, que são relevantes em canais de alta permeabilidade que surgem devido ao processo reacional.
\end{abstract}

\section{INTRODUÇÃO}

Com o objetivo de estimular a produção de óleo e gás, utiliza-se o processo de acidificação, no qual um determinado tipo ácido é injetado na rocha para reagir e dissolver parte dela, gerando um aumento na sua permeabilidade local. Um dos principais objetivos é o controle adequado da operação de forma a gerar canais de alta permeabilidade, chamados wormholes, que dependem de condições como a vazão de injeção, a heterogeneidade da rocha, a cinética reacional e a temperatura do reservatório (Maheshwari et al., 2013; Panga et al., 2005).

O Modelo de Duas Escalas Contínuas descrito por Panga et al. (2005) está entre os mais utilizados no desenvolvimento de metodologias numéricas para estudo da formação de wormholes e estimulação de meios porosos carbonáticos. Este modelo é baseado no uso da equação de Darcy para o transporte de quantidade de movimento, o qual despreza a relevância dos termos inerciais e difusivos no movimento do fluido. Contudo, devido ao aumento expressivo na permeabilidade local do meio gerado no processo de acidificação, desconsiderar esses termos no transporte de quantidade movimento é questionável (Dias, 2017).

Neste estudo é proposto a utilização da média volumétrica da equação de quantidade de movimento (VAE - Volume Average Equation), baseada no trabalho desenvolvido por Dias (2017). Os modelos foram numericamente implementados no código OpenFOAM®, o qual utiliza o método de volumes clássico com arranjo co-localizado das variáveis na malha e esquemas segregados de solução das equações de transporte baseados em algoritmos de acoplamento derivados do SIMPLE e do PISO (Ferziger e Perić, 1997; Moukalled et al., 2015). 


\section{EQUACIONAMENTO DOS MODELOS}

\section{1. $\quad$ Modelo de Darcy}

O modelo proposto por Panga et al. (2005) é baseado na modelagem de duas escalas: (i) a escala de Darcy e (ii) a escala de poro. A escala de Darcy é modelada pelas equações de continuidade 1, de quantidade de movimento linear (equação de Darcy) 2, transporte de espécie química 3 , reação heterogênea 4 e evolução temporal da porosidade 5 :

$$
\begin{gathered}
\frac{\partial(\rho \phi)}{\partial t}+\nabla \cdot(\rho \mathbf{U})=0, \\
\mathbf{U}=-\frac{1}{\mu} \mathbf{K} \cdot(\nabla p-\rho \mathbf{g}), \\
\frac{\partial\left(\phi C_{f}\right)}{\partial t}+\nabla \cdot\left(\mathbf{U} C_{f}\right)=\nabla \cdot\left(\phi \mathbf{D}_{e} \cdot \nabla C_{f}\right)-k_{c} a_{v}\left(C_{f}-C_{s}\right), \\
k_{c} a_{v}\left(C_{f}-C_{s}\right)=R\left(C_{s}\right)=k_{s} C_{s}, \\
\frac{\partial \phi}{\partial t}=\frac{R\left(C_{s}\right) a_{v} \alpha}{\rho_{s}}
\end{gathered}
$$

onde $\mathbf{U}$ é o campo de velocidades, $\mathbf{K}$ é o tensor permeabilidade, $p$ é a pressão, $C_{f}$ é a concentração de ácido na fase líquida, $C_{s}$ é a concentração de ácido na interface fluidosólido, $\mathbf{D}_{e}$ é o tensor de dispersão efetiva, $k_{c}$ é a coeficiente de transferência de massa, $a_{v}$ é a densidade de área interfacial do poro, $\rho_{s}$ é a densidade do sólido, $\alpha$ é o poder de dissolução do ácido e $R\left(C_{s}\right)$ é taxa de reação heterogênea e $k_{s}$ a constante de velocidade de reação de primeira ordem (Panga et al., 2005).

O fechamento do sistema de equações na escala de Darcy é feito através de relações estruturais e informações da interação fluido-sólido, que são provenientes de modelos na escala de poro, de acordo com as equações:

$$
\begin{gathered}
K=K_{0} \frac{\phi}{\phi_{0}}\left[\frac{\phi\left(1-\phi_{0}\right)}{\phi_{0}(1-\phi)}\right]^{2 \beta}, \\
\frac{a_{v}}{a_{0}}=\frac{\phi}{\phi_{0}} \frac{r_{p_{0}}}{r_{p}}, \quad \frac{r_{p}}{r_{0}}=\sqrt{\frac{K}{K_{0}} \frac{\phi_{0}}{\phi}}, \\
k_{c}=\frac{D_{m} S h}{2 r_{p}}, \quad S h=S h_{\infty}+b R e_{p}^{1 / 2} S c^{1 / 3} .
\end{gathered}
$$

sendo $S h$ o número de Sherwood, $D_{m}$ é a difusividade molecular do ácido, $S c$ o número de Schmidt, $R e_{p}$ é o número de Reynolds associado ao diâmetro de poro, $b$ é uma constante associada à estrutura do meio poroso e $S h_{\infty}$ é o número de Schmidt no bulk do escoamento.

O tensor dispersão $\mathbf{D}_{e}$ é diagonal e seus elementos são dados por:

$$
D_{e_{i}}=D_{m} \alpha_{0 s}+\lambda_{i} P e_{p}, \quad i \in\{X, Y, Z\}, \quad P e_{p}=\frac{2 r_{p}|\mathbf{U}|}{\phi D_{m}}
$$

onde $\alpha_{0 s}$ uma constante associada à tortuosidade do meio, $\lambda_{i}, i \in\{X, Y, Z\}$ são as constantes relativas à direção da dispersão e $P e_{p}$ o número de Peclet no poro (Panga et al., 2005). 


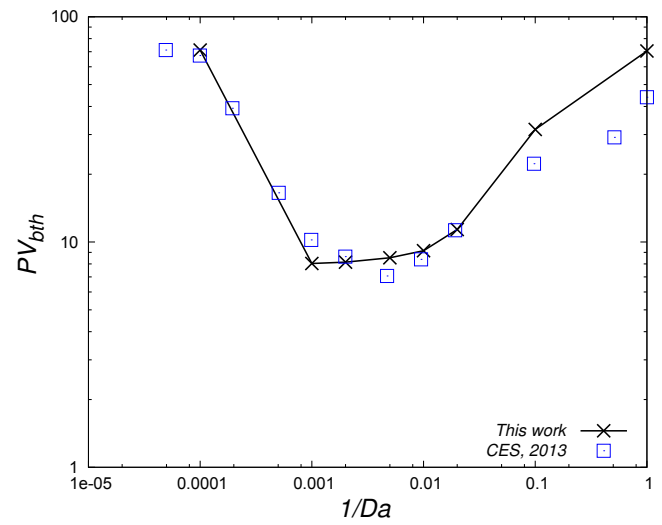

(a)

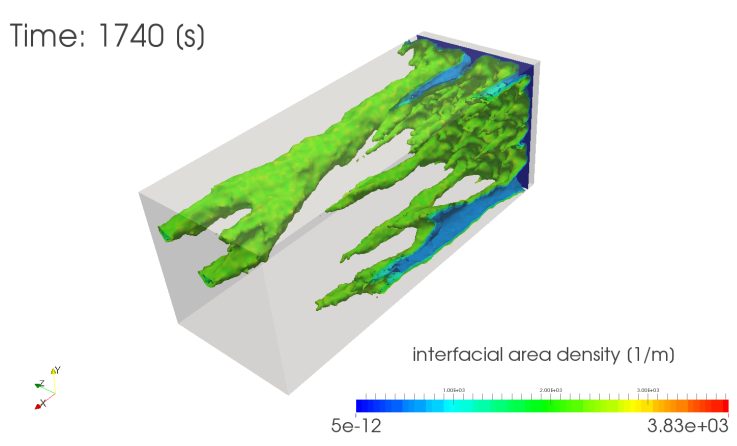

(b)

Figura 1 - Comparação entre resultados obtidos neste trabalho com os de Maheshwari et al. (2013) para o $P V_{b t h}$ (Pore Volume to breakthrough) na acidificação de plug retangular. (a) Gráfico de $P V_{b t h}$ variando com o número de Damköhler. (b) Perfil do meio acidificado no instante $t=1740[s]$ para $D_{a}=50$.

\subsection{Modelo VAE}

A diferença do modelo VAE proposto em relação ao modelo definido pelas Equações 1 a 5 reside no equacionamento da quantidade de movimento linear, que passa a ser definida pela Equação 10, obtida através da teoria de Média Volumétrica (Dias, 2017; Whitaker, 1999).

$$
\begin{aligned}
& \frac{\partial(\rho \phi \mathbf{U})}{\partial t}+\nabla \cdot(\rho \phi \mathbf{U} \otimes \mathbf{U})=-\phi \nabla p-\phi^{2} \mu^{e f f} \mathbf{D} \cdot \mathbf{U} \\
& +\rho \phi \mathbf{g}+\phi\left[\mu^{e f f}\left(\nabla \mathbf{U}+\nabla \mathbf{U}^{T}\right)-\frac{2}{3} \mu^{e f f} \nabla \cdot \mathbf{U}\right], \quad \text { com } \quad \mathbf{D}=\mathbf{K}^{-1}
\end{aligned}
$$

\section{RESULTADOS}

\subsection{Validação do Modelo VAE}

O trabalho feito por Maheshwari et al. (2013) foi reproduzido usando os seus mesmos parâmetros físicos e geométricos. A Figura 1 ilustra o resultado da comparação.

\subsection{Comparação Entre os Modelos}

A Figura 2 compara as curvas de $P V_{b t h}$ obtidas usando o modelo de Darcy e o modelo VAE usando mesmos dados geométricos e parâmetro físicos. Nota-se o maior afastamento dos resultados nas proximidades do ponto mínimo da curva de $P V_{b t h}$, região onde canais preferenciais de escoamento (wormholes) são predominantemente formados. 


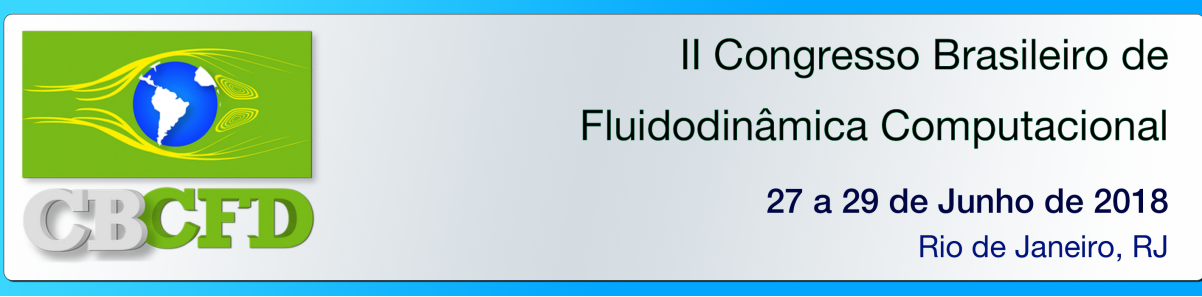

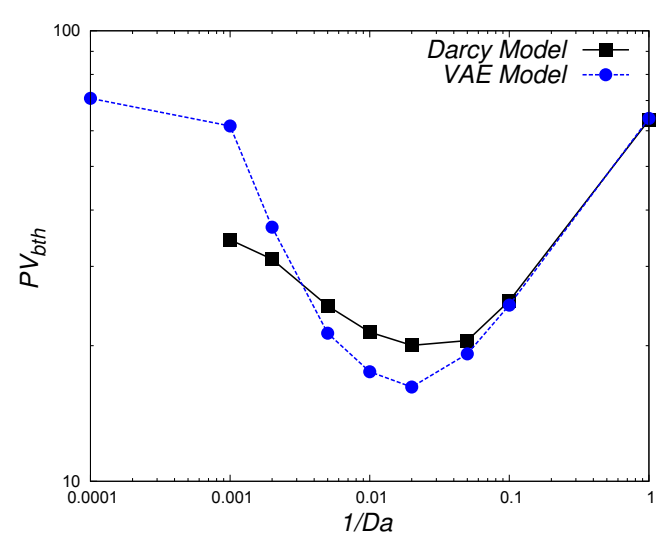

(a)

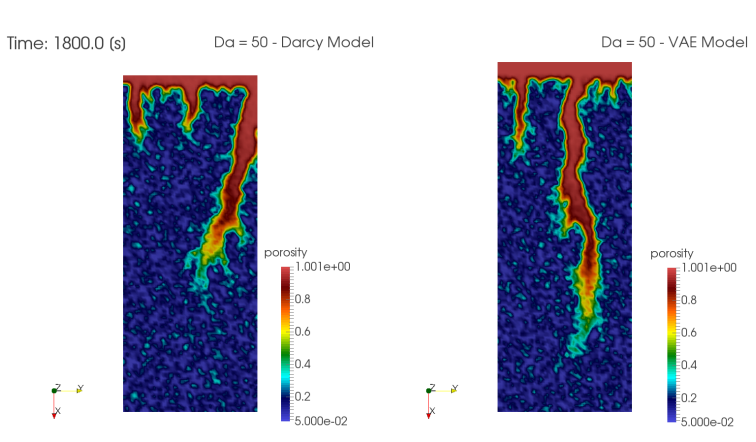

(b)

Figura 2 - Comparação entre resultados de acidificação usando o modelo de Darcy e modelo VAE. (a) Gráfico $P V_{b t h}$ variando com o número de Damköhler. (b) Perfil do meio acidificado no instante $t=1800[s]$ para $D_{a}=50$.

\section{CONCLUSÕES}

Os resultados apresentados neste trabalho mostraram que o modelo VAE é capaz de reproduzir o padrão esperado do processo de acidificação em plugs de rocha calcária. Além disso, pela comparação dos resultados do VAE com o modelo baseado na equação de Darcy, mostrou-se a relevância de se considerar os termos inerciais e difusivos na equação de quantidade de movimento linear, especialmente nas condições de operação favoráveis à formação de wormholes.

\section{REFERÊNCIAS}

DiAs, R. A. C. Produtividade de poços em reservatórios de alta permeabilidade. Tese de Doutorado, Pontifícia Universidade Católica, Rio de Janeiro, RJ, Brasil, 2017.

Ferziger, J. H.; Perić, M. Computational Methods for Fluid Dynamics. Springer, Germany, 2nd edition edição, 1997.

Maheshwari, P.; Ratnakar, R. R.; N, K.; Balakotaiah, V. 3-d simulation and analysis of reactive dissolution and wormhole formation in carbonate rocks. Chemical Engineering Science, 90, 258-274, 2013.

Moukalled, F.; Mangani, L.; Darwish, M. The Finite Volume Method in Computational Fluid Dynamics. Springer, 2015.

Panga, M. K. R.; Ziauddin, M.; Balakotaiah, V. Two-scale continuum model for simulation of wormholes in carbonate acidization. AIChE Journal, 51, 2005.

Whitaker, S. The Method of Volume Averaging. Springer Science + Business, 1999. 